\title{
SELF-INDUCTANCE OF A SOLENOID OF ANY NUMBER OF LAYERS.
}

\section{By Louis Cohen.}

The only formula heretofore available for the calculation of the self-inductance of a long coil or solenoid of more than one layer is that of Maxwell, which is as follows:

$$
L=\frac{4}{3} \pi^{2} n^{4} l(x-y)\left(x^{3}-y^{3}\right)
$$

where $l$ is the length of the solenoid, $n$ is the number of turns per unit length, $x$ and $y$ are the external and internal radii of the solenoid. This formula, however, was developed on the assumption of a uniform field within the coil, which means that the end effects can be neglected. The assumption may lead to an error as great as I 2 per cent as I shall show later, even in the case of a comparatively long solenoid, when the length is ten times the radius. It is quite evident that such a formula is not of very great value where accurate results are desired. It is, however, a very simple and convenient formula for numerical computations and is useful when only a rough approximation is desired.

I propose to develop in the following pages a formula for the selfinductance of a solenoid of any number of layers, and which will give results accurate to within one-half of I per cent even for a short solenoid, where the length is only twice the diameter, the accuracy increasing as the length increases. For most practical cases this degree of accuracy is amply sufficient.

Suppose we have a solenoid of $m$ layers, the mean radius of the first layer being $a_{1}$, and of the last layer $a_{m}$, then the self-inductance of the solenoid will be given by the following expression: 


$$
\begin{aligned}
L= & L_{1}+L_{2}+L_{3}+\ldots \ldots+L_{m} \\
& +2\left[M_{12}+M_{13}+\ldots \ldots+M_{1 m}\right. \\
& +M_{23}+M_{24}+\ldots \ldots+M_{2 m} \\
& +M_{34}+M_{35}+\ldots \ldots+M_{3 m} \\
& +\ldots \ldots \ldots \\
& +\ldots \ldots \ldots \ldots
\end{aligned}
$$

$L_{1}, L_{2}, L_{3}$, etc., are the self-inductances of single layer solenoids whose inean radii are $a_{1}, a_{2}, a_{3}$, etc., and the $M s$ are the mutual inductances between the various single layer solenoids. We have various formulae for the self-inductance of a single layer solenoid, and also for the mutual inductance of two coaxial solenoids, ${ }^{1}$ and if after substituting the various values for $L$ and $M$, we can combine them so as to give a simple expression, we shall evidently have a useful formula for the self-inductance of a solenoid of $m$ layers.

In a previous paper ${ }^{2} \mathrm{I}$ have developed an absolute formula for the self-inductance of a single layer solenoid, which is as follows:

$$
L=4 \pi n^{2}\left\{\frac{l^{4}+4 a^{2} l^{2}}{3 \sqrt{4 a^{2}+l^{2}}} F+\frac{4 a^{2}-l^{2}}{3} \sqrt{4 a^{2}+l^{2}} E-\frac{8 a^{3}}{3}\right\}
$$

$a$ is the mean radius, $l$ is the length of the solenoid, and $n$ is the number of turns of wire per centimeter, $F$ and $E$ are the complete elliptic integrals of the first and second kind to modulus $k$, where

$$
k^{2}=\frac{4 a^{2}}{4 a^{2}+l^{2}}
$$

This formula was first given by Lorenz without, however, giving its derivation. $F$ and $E$ can be put in the form of series, thus:

$$
\begin{aligned}
& F=\frac{\pi}{2}\left\{\mathrm{I}+\frac{\mathrm{I}}{2^{2}} k^{2}+\frac{\mathrm{I} \cdot \cdot^{2} 3^{2}}{2 \cdot^{2} 4^{2}} k^{4}+\ldots\right\} \\
& \left.E=\frac{\pi}{2} \mid \mathrm{I}-\frac{\mathrm{I}}{2^{2}} k^{2}-\frac{\mathrm{I} \cdot{ }^{2} 3}{2 \cdot \cdot^{2} 4^{2}} k^{4}-\ldots\right\}
\end{aligned}
$$

1 This Bulletin, :3, p. 305 .

${ }^{2}$ This Bulletin, 3, p. $3 \circ 3$. 
If $l=4 a$, then $k^{2}=\frac{4}{20}=\frac{I}{5}$, and the term in $k^{4}$ will be only about $\frac{I}{200}$ which may be neglected if we are aiming at an accuracy of only one half of a per cent. For longer solenoids the accuracy will of course be higher. Using, therefore, only the first two terms of the above series, and introducing the value of $k^{2}$, we get:

$$
\begin{aligned}
& F=\frac{\pi a^{2}+l^{2}}{2} \frac{4 a^{2}+l^{2}}{2} \\
& E=\frac{\pi}{2} \frac{3 a^{2}+l^{2}}{4 a^{2}+l^{2}}
\end{aligned}
$$

Introducing the values of $F$ and $E$ into equation (3) and simplifying we obtain

$$
L=4 \pi^{2} n n^{2}\left\{\frac{2 a^{4}+a^{2} l^{2}}{\sqrt{4 a^{2}+l^{2}}}-\frac{8}{3} \frac{a^{3}}{\pi}\right\}
$$

This is a very simple and convenient approximate formula for the calculation of the self-inductance of a single layer solenoid.

The self-inductance of a solenoid is a function of its radius, hence if we denote by $L_{0}$ the self-inductance of the central layer solenoid, the self-inductance of the various other layers will be given by the following formulæ:

$$
\begin{aligned}
& L_{\frac{m}{2}-1}=L_{0}-\int_{a_{\frac{m}{2}}}^{a_{0}} \frac{d L}{d \alpha} d a \\
& L_{\frac{m-2}{2}}=L_{0}-\int_{a_{\frac{m}{2}}}^{a_{0}} \frac{d L}{d a} d a-\int_{\frac{a_{m}-1}{2}}^{a_{\frac{m}{2}}} \frac{d L}{d \alpha} d a \\
& L_{\frac{m}{2}+1}=L_{0}+\int_{a_{0}}^{\frac{a_{m}}{2}} \frac{d L}{d a} d a \\
& L_{\frac{m}{2}+2}=L_{0}+\int_{a_{0}}^{a_{\frac{m}{2}}^{a_{2}}} \frac{d L}{d x} d a+\int_{a_{\frac{m}{z}}}^{a_{\frac{m}{2}}+1} d L d a
\end{aligned}
$$


Usually the winding is of comparatively fine wire, say, O.I to 0.2 c111., and therefore the value of any of the above integrals will be a small quantity as compared with $\mathrm{L}_{0}$, and the difference between any two of the above integrals will be an exceedingly small quantity. In summing up the self-inductances of the various layers, we evidently hare as many negative as positive integrals which will nearly cancel each other and therefore:

$$
\Sigma L=m L_{0}=m 4 \pi^{2} l^{2}\left\{\frac{2 a_{0}^{4}+a_{0}^{2} l^{2}}{\sqrt{4 a_{0}^{2}+l^{2}}}-\frac{8 a_{0}^{3}}{3 \pi}\right\}
$$

when $a_{0}$ is the mean radius of the solenoid.

It remains now to find the value of $B$ of equation (2). The nuntual inductance between two coaxial solenoids is given by the following expression ${ }^{3}$

$$
M=4 \pi^{2} n^{2}[1-2 \cdot A a]
$$

where

$$
a=\frac{l-r+A}{2 A}-\frac{a^{2}}{\mathrm{I} 6 \cdot A^{4}}\left(\mathrm{I}-\frac{A^{3}}{r^{3}}\right)-\frac{a^{4}}{64 A^{4}}\left(\mathrm{I} / 2+\frac{2 \cdot A^{5}}{r^{5}}-\frac{5 A^{7}}{2 r^{i}}\right)+\ldots
$$

$r=\sqrt{A^{2}+l^{3}}, A$ is the radius of the external solenoids, and $a$ is the radius of the internal solenoid. Since, however, we are only aiming at an accuracy of one-half per cent for short solenoids we may put

$$
a=\frac{1-r+.4}{2.1}-\frac{a^{2}}{16 \cdot A^{2}}
$$

and neglect the other terms. Introducing this value of $a$ we get:

$$
M=+\pi^{2} a^{2} n^{2}\left\{\sqrt{A^{2}+l^{2}}-A+\frac{a^{2}}{S A}\right\}
$$

Putting $a_{2}=a_{1}+\delta a, a_{3}=a_{1}+2 \delta a_{1} \ldots \ldots a_{m}=a_{1}+(m-\mathrm{I}) \delta a$ where $\delta a$ is the distance between two consecutive layers, and also expanding the term nnder the radical and neglecting small terms, we shall have: 


$$
\begin{aligned}
& M_{1,2}=4 \pi^{2} a_{1}^{2} n^{2}\left\{\sqrt{a_{1}^{2}+l^{2}}+\frac{a_{1} \delta a}{\sqrt{a_{1}^{2}+l^{2}}}-a_{1}-\delta a+\frac{a_{1}}{8}-\frac{\delta a}{8}\right\} \\
& M_{1.3}=4 \pi^{2} a_{1}^{2} n^{2}\left\{\sqrt{a_{1}^{2}+l^{2}}+\frac{2 a_{1} \delta a}{\sqrt{a_{1}^{2}+l^{2}}}-a_{1}-2 \delta a+\frac{a_{1}}{8}-\frac{2 \delta a}{8}\right\}
\end{aligned}
$$

$$
\begin{aligned}
M_{1, m}=4 \pi^{2} a_{1}^{2} n^{2}\left\{\sqrt{a_{1}^{2}+l^{2}}\right. & +\frac{(m-\mathrm{I}) a_{1} \delta a}{\sqrt{a_{1}^{2}+l^{2}}-a_{1}-(m-\mathrm{I}) \delta a+\frac{a_{1}}{8}} \\
& \left.-\frac{(m-\mathrm{I}) \delta a}{8}\right\}
\end{aligned}
$$

$$
M_{2,3}=4 \pi^{2} a_{2}^{2} n^{2}\left\{\sqrt{a_{1}^{2}+l^{2}}+\frac{2 a_{1} \delta a}{\sqrt{a_{1}^{2}+l^{2}}}-a_{1}-2 \delta a+\frac{a_{1}}{8}\right\}
$$$$
M_{2,4}=4 \pi^{2} a_{2}^{2} n^{2}\left\{\sqrt{a_{1}^{2}+l^{2}}+\frac{3 a_{1} \delta a}{\sqrt{a_{1}^{2}+l^{2}}}-a_{1}-3 \delta a+\frac{\alpha_{1}}{8}-\frac{\delta a}{8}\right\}
$$$$
M_{2 . m}=4 \pi^{2} a_{2}^{2} n^{2}\left\{\sqrt{a_{1}^{2}+l^{2}}+\frac{(m-\mathrm{I}) \delta a}{\sqrt{a_{1}^{2}+l^{2}}}-a_{1}-(m-\mathrm{I}) \delta a+\frac{a_{1}}{8}-\frac{(m-3) \delta a}{8}\right\}
$$$$
M_{3,4}=4 \pi^{2} a_{3}^{2} n^{2}\left\{\sqrt{a_{1}^{2}+l^{2}}+\frac{3 a_{1} \delta a}{\sqrt{a_{1}^{2}+l^{2}}}-a_{1}-3 \delta a+\frac{a_{1}}{8}+\frac{\delta a}{8}\right\}
$$$$
M_{3,5}=4 \pi^{2} a_{3}^{2} n^{2}\left\{\sqrt{a_{1}^{2}+l^{2}}+\frac{4 a_{1} \delta a}{\sqrt{a_{1}^{2}+l^{2}}}-a_{1}-4 \delta a+\frac{a_{1}}{8}\right\}
$$

$$
\begin{gathered}
M_{3, m}=4 \pi^{2} a_{3}^{2} n^{2}\left\{\sqrt{a_{1}^{2}+l^{2}}+\frac{(m-\mathrm{I}) \delta a}{\sqrt{a_{1}^{2}+l^{2}}}-a^{2}-(m-\mathrm{I}) \delta a\right. \\
\left.+\frac{a_{1}-\frac{(m-5)}{8} \delta a}{8}\right\} \\
M_{4, m}=4 \pi^{2} a_{4}^{2} n^{2}\left\{\sqrt{a_{1}^{2}+l^{2}}+\frac{(m-\mathrm{I}) a_{1} \delta a}{\sqrt{a_{1}^{2}+l^{2}}}-a_{1}-(m-\mathrm{I}) \delta a+\frac{a_{1}}{8}\right. \\
\left.-\frac{(m-7)}{8} \delta a\right\}
\end{gathered}
$$




$$
\begin{gathered}
M_{m-1, m}=4 \pi^{2} a^{2}{ }_{m-1} n^{2}\left\{\sqrt{a_{1}^{2}+l^{2}}+\frac{(m-\mathrm{I}) a_{1} \delta a}{\sqrt{a_{1}^{2}+l^{2}}-a_{1}-(m-\mathrm{I}) \delta a+\frac{a_{1}}{8}}\right. \\
\left.-\frac{(m-2 m+\mathrm{I})}{8} \delta a\right\}
\end{gathered}
$$

Summing up we get:

$$
\begin{aligned}
& M_{1,2}+M_{1,3}+\ldots+M_{1 . m}=4 \pi^{2} a_{1}^{2} n^{2}(m-\mathrm{I})\left\{\sqrt{a_{1}^{2}+l^{2}}+\frac{m a_{1} \delta a}{2 \sqrt{a_{1}^{2}+l^{2}}}\right. \\
& \left.-a_{1}-\frac{m}{2} \delta a+\frac{a_{1}}{8}-\frac{m}{2} \frac{\delta a}{8}\right\} \\
& M_{23}+M_{24}+\ldots \ldots+M_{2, m}=4 \pi^{2} a_{2}^{2} n^{2}(m-2)\left\{\sqrt{a_{1}^{2}+l^{2}}+\frac{(m+\mathrm{I}) a_{1} \delta a}{2 \sqrt{a_{1}^{2}+l^{2}}}-a_{1}\right. \\
& \left.-\frac{(m+\mathrm{I}) \delta a}{2}+\frac{a_{1}}{8}-\frac{(m-3)}{2} \frac{\delta a}{8}\right\} \\
& M_{3,4}+M_{3,5}+\cdots+M_{3, m}=4 \pi^{2} \alpha_{3}^{2} n^{2}(m-3)\left\{\sqrt{a_{1}^{2}+l^{2}}+\frac{(m+2) a_{1} \delta a}{2 \sqrt{a_{1}^{2}+l^{2}}}\right. \\
& \left.-a_{1}-\frac{(m+2) \delta a}{2}+\frac{a_{1}}{8}-\frac{(m-4)}{2} \frac{\delta a}{8}\right\} \\
& M_{4,5}+M_{4,6}+\ldots+M_{4, m}=4 \pi^{2} a_{4}^{2} n^{2}(m-4)\left\{\sqrt{a_{1}^{2}+l^{2}}+\frac{(m+3) a_{1} \delta a}{2 \sqrt{a_{1}^{2}+l^{2}}}-\right. \\
& \left.a_{1}-\frac{(m+3)}{2} \delta a+\frac{a_{1}}{8}-\frac{(m-5)}{2} \frac{\delta a}{8}\right\}
\end{aligned}
$$

Adding all the summation terms we get for the total mutual inductance between the various layers:

$$
\begin{aligned}
& \Sigma M=4 \pi^{2} n^{2}\left\{[ ( m - \mathrm { I } ) a _ { 1 } ^ { 2 } + ( m - 2 ) a _ { 2 } ^ { 2 } + ( m - 3 ) a _ { 3 } { } ^ { 2 } + \ldots ] \left(\sqrt{a_{1}{ }^{2}+l^{2}}\right.\right. \\
& \left.-\frac{7}{8} a_{1}\right)+\frac{\mathrm{I}}{2}\left[m(m-\mathrm{I}) a_{1}^{2}+(m-\mathrm{I})(m-2) a_{2}^{2}+(m-2)(m-3) a_{3}^{2}\right. \\
& \left.+\ldots . .]\left(\frac{a_{1} \delta a}{\sqrt{a_{1}^{2}+l^{2}}}-\delta a\right)-\frac{\mathrm{I}}{2}\left[m(m-\mathrm{I}) a_{1}^{2}+(m-2)(m-3) a_{2}^{2}+\ldots\right] \frac{\delta a}{8}\right\}
\end{aligned}
$$


The final formula for the self-inductance of a solenoid of $m$ layers will therefore be as follows:

$$
\begin{gathered}
L=4 \pi^{2} n^{2} m\left\{\frac{2 a_{0}^{4}+a_{0}^{2} l^{2}}{\sqrt{4 a_{0}^{2}+l^{2}}}-\frac{8 a_{0}^{3}}{3 \pi}\right\}+8 \pi^{2} n^{2}\left\{\left[(m-\mathrm{I}) a_{1}^{2}+(m-2) a_{2}^{2}+\ldots\right]\right. \\
\left(\sqrt{a_{1}^{2}+l^{2}}-\frac{7}{8} a_{1}\right)+\frac{\mathrm{I}}{2}\left[m(m-\mathrm{I}) a_{1}^{2}+(m-\mathrm{I})(m-2) a_{2}^{2}+\ldots\right] \\
\left(\sqrt{\left.\left.\frac{a_{1} \delta a}{a_{1}^{2}+l^{2}}-\delta a\right)-\frac{\mathrm{I}}{2}\left[m(m-\mathrm{I}) a_{1}{ }^{2}+(m-2)(m-3) a_{2}{ }^{2}+\ldots\right] \frac{\delta a}{8}\right\} \quad(7)}\right.
\end{gathered}
$$

The last term in equation (7) is only about $\mathrm{I} / 500$ of the total inductance even in the case of a relatively short solenoid, where the length is twice the diameter, and hence we may neglect the last term. We therefore have:

$$
\begin{aligned}
L= & 4 \pi^{2} n^{2} m\left\{\frac{2 a_{0}^{4}+a_{0}^{2} l^{2}}{\sqrt{4 a_{0}^{2}+l^{2}}}-\frac{8 a_{0}^{3}}{3 \pi}\right\}+8 \pi^{2} n^{2}\left\{\left[(m-\mathrm{I}) a_{1}^{2}+(m-2) a_{2}^{2}+\ldots\right]\right. \\
& \left.\left(\sqrt{a_{1}^{2}+l^{2}}-\frac{7}{8} a_{1}\right)+\frac{\mathrm{I}}{2}\left[m(m-\mathrm{I}) a_{1}^{2}+\ldots\right]\left(\frac{a_{1} \delta a}{\sqrt{a_{1}^{2}+l^{2}}}-\delta a\right)\right\}
\end{aligned}
$$

For long solenoids, where the length is, say, four times the diameter, we can neglect also the last term in equation (8).

The formula for the self-inductance as given by equation (8) is a simple one and very convenient for numerical computations.

Below are two numerical examples to show the per cent of error that Maxwell's formula (I) will introduce:

\section{EXAMIPLE 1.}

$$
a_{1}=5 \mathrm{~cm}, \delta a=\text { O.I cm, } m=4, a_{0}=5.15 \mathrm{~cm}, l=50 \mathrm{~cm} \text {. }
$$

Introducing these values in $(\tau)$ we get:

$$
L=4 \pi^{2} n^{2}\{484 \text { I.9 }+ \text { I } 4 \text { I } 34.7-45.9-4.4\}=4 \pi^{2} n^{2} \times \text { I } 8926
$$

It is seen that in this case where the length is five times the diameter, even the third term of equation $(7)$ is only about one fourhundredth part of the total inductance, which may be neglected in approximate calculations. 
The same example calculated by Maxwell's formula gives

$$
L=4 \pi^{2} n^{2} \times 21228
$$

The error is a little over 12 per cent.

EXAMPLE 2.

$$
A_{1}=5 \mathrm{~cm}, \delta a=0.2 \mathrm{~cm}, m=5, a_{0}=5.4 \mathrm{~cm}, l=20
$$

By formula (7)

$$
L=4 \pi^{2} n^{2}\{227 \mathrm{I} .5+8795 \cdot 9-\mathrm{I} 6 \mathrm{I} \cdot 5-\mathrm{I} 8\}=4 \pi^{2} n^{2} \times \mathrm{IO} 888
$$

By Maxwell's formula (I) we get for this case:

$$
L=4 \pi^{2} n^{2} \times 14622
$$

The error in formula ( $\mathrm{I}$ ) is therefore about 35 per cent.

WaShington, October II, 1907. 